\title{
Epidemiología de la fractura de cadera en la Argentina
}

\author{
Germán Garabano, Adriana Cubecino, Harold Simesen de Bielke, Nicolás Robador, \\ Javier M. Olivetto, Mónica Sierto, Débora Gamarra \\ Comité de Morbi-Mortalidad de la Asociación Argentina de Ortopedia y Traumatología, \\ Ciudad Autónoma de Buenos Aires, Argentina
}

\section{RESUMEN}

La Asociación Argentina de Ortopedia y Traumatología a través del Comité de Morbi-Mortalidad confeccionó una encuesta sobre fracturas de cadera, con el fin de caracterizar este cuadro en nuestro país. Al completarse las primeras 1000 encuestas se elaboró este informe descriptivo. Se estima que, en la Argentina, la prevalencia de fracturas de cadera es de aproximadamente 264/100.000 habitantes. Estas lesiones resultaron más frecuentes en mujeres que en hombres (relación 2,7:1). El 80\% de los pacientes tenía >70 años y múltiples comorbilidades (hipertensión arterial, diabetes, anemia y osteoporosis, entre las más frecuentes). El $60 \%$ deambulaba solo dentro del domicilio. La incidencia de fracturas intertrocantéricas y mediales fue similar: $53 \%$ y $47 \%$, respectivamente. La tasa de complicaciones posoperatorias fue de aproximadamente el 13\%, y la infección fue la complicación más frecuente. La tasa de mortalidad en los primeros meses posteriores a la cirugía osciló entre el 3,8\% y el 4,85\%.

Palabras clave: Fractura de cadera; morbilidad; mortalidad; prevalencia; epidemiología.

Nivel de Evidencia: IV

\section{Epidemiology of hip fractures in Argentina}

\section{ABSTRACT}

The Morbidity and Mortality Committee of the Argentine Association of Orthopedics and Traumatology draw up a structured questionnaire on hip fractures, in order to characterize this pathology in Argentina. After receiving data on the first 1000 cases, we prepared this descriptive report. In Argentina, the estimated hip fracture prevalence is approximately 264/100,000 inhabitants. These injuries were more frequent in women, with a female-to-male ratio of 2.7:1. Eighty percent of patients were older than 70 years and presented with multiple comorbidities (the most common being high-blood pressure, diabetes, anemia, and osteoporosis). Sixty percent of patients were able to walk indoors independently. The incidence of intertrochanteric and femoral neck fractures was $53 \%$ and $47 \%$, respectively. The postoperative complication rate was approximately $13 \%$, infection being the most common. The mortality rate during the first months ranged between $3.8 \%$ and $4.85 \%$.

Key words: Hip fracture; morbidity; mortality; prevalence; epidemiology.

Level of Evidence: IV

\section{INTRODUCCIÓN}

Las fracturas de cadera generan un importante impacto en la morbimortalidad de los pacientes, sobre todo, en los adultos mayores, y representan un problema de salud pública en el mundo.

En nuestro país, si bien no contamos con estadísticas gubernamentales representativas, de acuerdo con un informe de 2013 sobre la población latinoamericana, la incidencia de fracturas de cadera era de aproximadamente 264 cada 100.000 habitantes en la Argentina. ${ }^{1}$ En un estudio reciente (2020) sobre fracturas de cadera en Latinoamérica, la incidencia anual fue de 298 cada 100.000 mujeres y de 118 cada 100.000 hombres. $^{2}$ 
Como sucede en el resto del mundo, hace décadas que la base de la pirámide poblacional de nuestro territorio se va angostando, aumenta la expectativa de vida y, con ello, el envejecimiento de la población. ${ }^{3}$ En consecuencia, es esperable que la incidencia de estas fracturas se siga incrementando.

Desde hace algunos años, la Asociación Argentina de Ortopedia y Traumatología a través de su Comité de Morbi-Mortalidad ha estado elaborando una serie de encuestas para evaluar diferentes enfermedades prevalentes incluidas en nuestro accionar como traumatólogos, entre ellas, las fracturas de cadera. Esta encuesta fue confeccionada mediante el trabajo conjunto del Comité de Morbi-Mortalidad con la Asociación Argentina de Ortopedia y Traumatología, la Asociación Argentina para el Estudio de la Cadera y Rodilla (ACaRo) y la Asociación Argentina de Trauma Ortopédico (AATO). Los socios de la Asociación Argentina de Ortopedia y Traumatología la pueden consultar en la página web (https://aaot.org.ar/certificacion/comite-de-morbi-mortalidad/).

En la encuesta, se evalúan múltiples aspectos del paciente que sufre una fractura de cadera, entre ellos, las características demográficas, el nivel de actividad física anterior a la fractura, las condiciones de vida y las comorbilidades.

Se destacan también las variables prequirúrgicas (hematocrito, hemoglobina, ASA) y el tipo de fractura, las condiciones de internación (total de días, días de espera para la cirugía, etc.), el tipo de tratamiento, las complicaciones y la tasa de mortalidad, entre otras características.

Hasta diciembre de 2019 inclusive, se completaron 1000 encuestas. Se realizó el análisis de toda la información y se procedió a la redacción del informe, dividido en dos partes. En la primera (la presente), exponemos un análisis descriptivo de los datos demográficos, las condiciones prequirúrgicas, los tipos de fractura y los tratamientos, las complicaciones y la tasa de mortalidad registradas. En la segunda, se expondrá el análisis estadístico comparativo, entre los tipos y subtipos de fractura, el tratamiento indicado en cada una de ellas y sus respectivas complicaciones.

\section{INFORME (PRIMERA PARTE)}

Las respuestas a las encuestas fueron suministradas desde 34 centros de salud de diferentes regiones del país. Del total de 1000 encuestas, 597 (59,7\%) se originaron en el sistema público de salud y 403 (40,3\%), en el ámbito privado.

\section{Características demográficas}

La incidencia por género mostró que 735 (73,5\%) fracturas se produjeron en mujeres y 265 (26,5\%), en varones, es decir, una relación 2,7:1. La distribución, según la edad, se detalla en la Figura 1. Se destaca que el $80 \%$ tenía $>70$ años y el $51 \%,>80$ años.

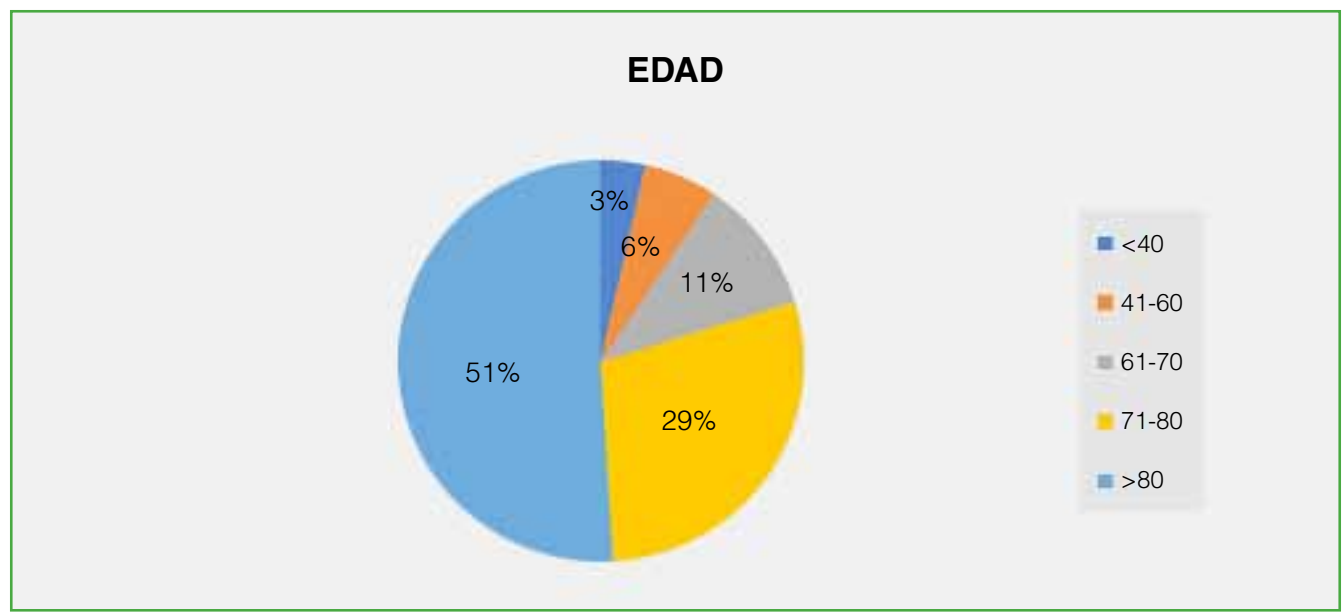

Figura 1. Distribución de las fracturas de cadera, según la edad. 


\section{Condiciones de vida y nivel de actividad antes de la fractura}

Respecto a la situación de vivienda de los pacientes, se observó que tres cuartos viven acompañados y que aproximadamente el $20 \%$ reside en una institución.

En cuanto al nivel de actividad antes de la fractura, más de la mitad deambulaba dentro del domicilio exclusivamente y, por otro lado, más de un tercio necesitaba de asistencia para la marcha (Tabla 1).

Tabla 1. Condiciones de vida y capacidad para deambular antes de la fractura

\begin{tabular}{|l|c|c|c|c|}
\hline & Vive solo & Institucionalizado & $\begin{array}{c}\text { Deambula dentro } \\
\text { del domicilio }\end{array}$ & $\begin{array}{c}\text { Necesita asistencia } \\
\text { para la marcha }\end{array}$ \\
\hline Sí n (\%) & $243(24,3)$ & $196(19,6)$ & $589(58,9)$ & $366(36,6)$ \\
\hline No n (\%) & $757(75,7)$ & $794(7,94)$ & $411(41,1)$ & $634(63,4)$ \\
\hline
\end{tabular}

\section{Comorbilidades}

Las comorbilidades más frecuentes de la serie fueron hipertensión, osteoporosis, insuficiencia cardíaca y diabetes (Tabla 2).

Tabla 2. Comorbilidades de los pacientes en el momento de la fractura

\begin{tabular}{|l|c|}
\hline Comorbilidades & n $(\%)$ \\
\hline Hipertensión arterial & $671(67,1)$ \\
\hline Osteoporosis & $178(17,8)$ \\
\hline Diabetes (1 y 2) & $117(11,7)$ \\
\hline Insuficiencia cardíaca & $116(11,6)$ \\
\hline Gastritis & $87(8,7)$ \\
\hline Demencia & $80(8)$ \\
\hline Enfermedad de Parkinson & $60(6)$ \\
\hline Insuficiencia renal & $47(4,7)$ \\
\hline Insuficiencia urinaria & $32(3,2)$ \\
\hline Alergia & $24(2,4)$ \\
\hline Artritis reumatoide & $23(2,3)$ \\
\hline Hipotiroidismo & $23(2,3)$ \\
\hline Asma & $16(1,6)$ \\
\hline Accidente cerebrovascular & $14(1,4)$ \\
\hline Enfermedad pulmonar obstructiva crónica & $13(1,3)$ \\
\hline Úlcera gástrica & $12(1,2)$ \\
\hline Tabaquismo & $10(1)$ \\
\hline Depresión & $6(0,6)$ \\
\hline Obesidad & $2(0,2)$ \\
\hline Otras: arritmia, hipertiroidismo, glaucoma, cáncer & \\
\hline
\end{tabular}




\section{Mecanismo de la lesión}

En una búsqueda por caracterizar el trauma que generó la fractura, se lo dividió en tres grupos de acuerdo con la energía: alta (p. ej., accidente de tránsito), mediana (p. ej., caída en bicicleta) y baja (p. ej., caída de propia altura). Hubo un amplio predominio del trauma de baja energía (Figura 2).

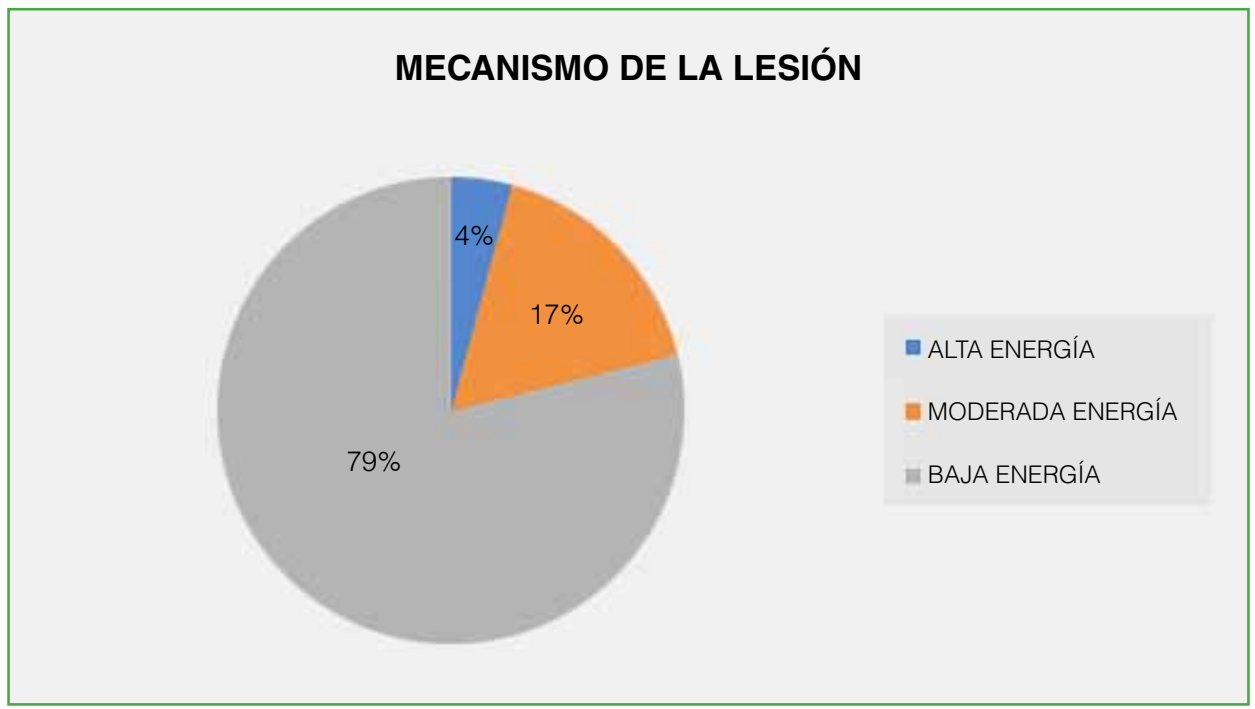

Figura 2. Tipo de trauma que generó la fractura.

\section{Tipo y subtipo de fracturas}

Las encuestas utilizan dos clasificaciones para caracterizar las fracturas: la clasificación de Garden para las mediales y la propuesta por Evans para las laterales.

Se excluyeron 51 de las 1000 respuestas $(5,1 \%)$, porque las fracturas estaban clasificadas de manera incorrecta (21 no tenían clasificación, y 30 eran mediales y laterales - doble clasificación-). En consecuencia, quedaron 949 fracturas para el análisis. De estas, $505(53,21 \%)$ correspondían a fracturas laterales o intertrocantéricas y 444 $(46,78 \%)$, a mediales. Los subtipos de cada una se detallan en las Figuras 3 y 4.

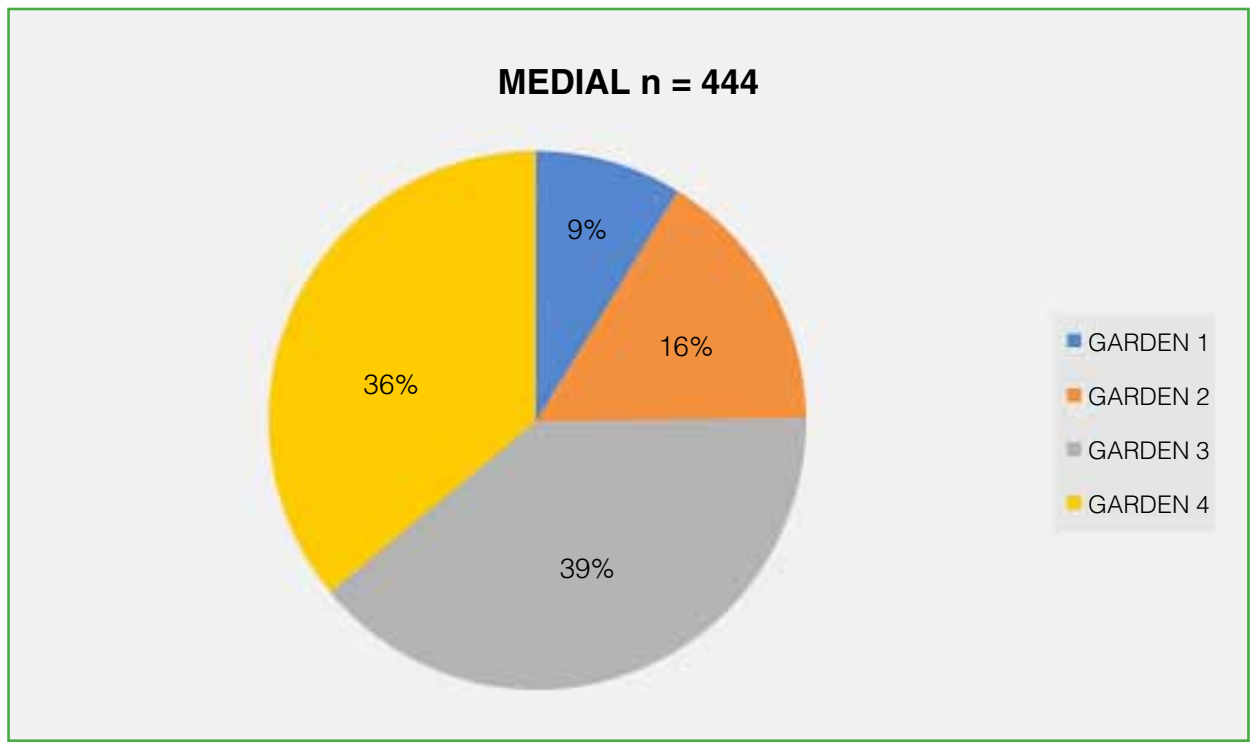

Figura 3. Subtipos de fracturas mediales según la clasificación de Garden. 


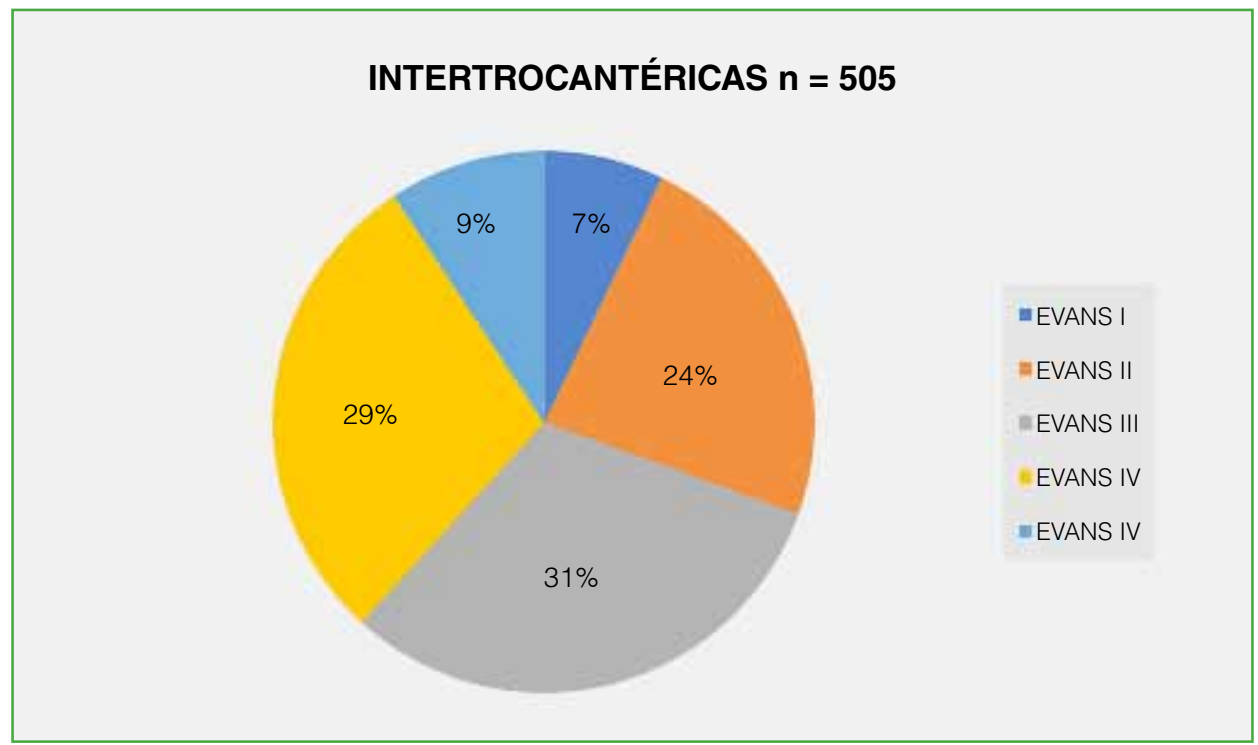

Figura 4. Subtipos de fracturas intertrocantéricas según la clasificación de Evans.

\section{Condición preoperatoria}

Las variables preoperatorias que se analizaron fueron: hematocrito, hemoglobina y el puntaje de la American Society of Anesthesiologists (ASA) como factor predictivo del riesgo quirúrgico. Según lo establecido, los grados I y II se consideraron de bajo riesgo quirúrgico; y los grados III y IV, de alto riesgo. Entre 844 respuestas sobre el puntaje ASA, el 57,58\% (486 pacientes) pertenecían al grupo de bajo riesgo y el 42,41\% (372 casos), al de alto riesgo. El hematocrito promedio era del 33,3\% (rango 19,7-42) sobre un total de 949 respuestas. La hemoglobina promedio era de 12,95 mg/dl (rango 6,5-19). Tomando como límite normal una hemoglobina de $12 \mathrm{mg} / \mathrm{dl}$, el $49,6 \%$ de los pacientes tenía anemia.

\section{Características de la internación}

Se registraron los días de espera para la cirugía (desde la admisión hasta el procedimiento quirúrgico) y la estancia hospitalaria completa (desde la admisión hasta el egreso). Los resultados mostraron que el 45,28\% de los pacientes con fracturas habían sido operados antes del sexto día de la internación y más del $25 \%$, luego del décimo día. Respecto a la estancia hospitalaria, la mitad permaneció internado 10 días o menos (Tabla 3).

Tabla 3. Descripción de la estancia hospitalaria

\begin{tabular}{|c|c|c|}
\hline Días & $\begin{array}{c}\text { Espera para la cirugía } \\
(901 \text { respuestas }) \\
\text { n(\%) }\end{array}$ & $\begin{array}{c}\text { Internación total } \\
(\mathbf{9 1 5} \text { respuestas }) \\
\mathbf{n}(\%)\end{array}$ \\
\hline $0-5$ & $408(45,28)$ & $190(20,76)$ \\
\hline $6-10$ & $265(29,41)$ & $304(33,22)$ \\
\hline $11-20$ & $169(18,75)$ & $295(32,24)$ \\
\hline$>20$ & $59(6,54)$ & $126(13,77)$ \\
\hline
\end{tabular}




\section{TRATAMIENTO}

El tratamiento se divide en dos grandes grupos: osteosíntesis y artroplastia. Las opciones de osteosíntesis representadas en las encuestas son cuatro: tornillos canulados, clavos cefalomedulares, tornillo placa deslizante y otros.

Las opciones para las artroplastias ofrecidas fueron tres: unipolar, bipolar, total. Para este último grupo, también se detalla el uso de cemento con antibióticos o sin ellos. En ambos grupos, se discrimina el origen del implante, nacional o importado, y el empleo o no de drenaje posoperatorio.

Sobre 444 respuestas para las fracturas mediales, el 77,25\% habían sido tratadas con artroplastia; el 22,29\%, con osteosíntesis y el $0,45 \%$, con artroplastia por resección (Girdlestone) (1 caso) y con tratamiento conservador (1 caso).

Las osteosíntesis y las artroplastias eran de origen nacional en el 91,08\% y el 88,04\%, respectivamente. Los diferentes tipos de tratamientos se detallan en la Figura 5.

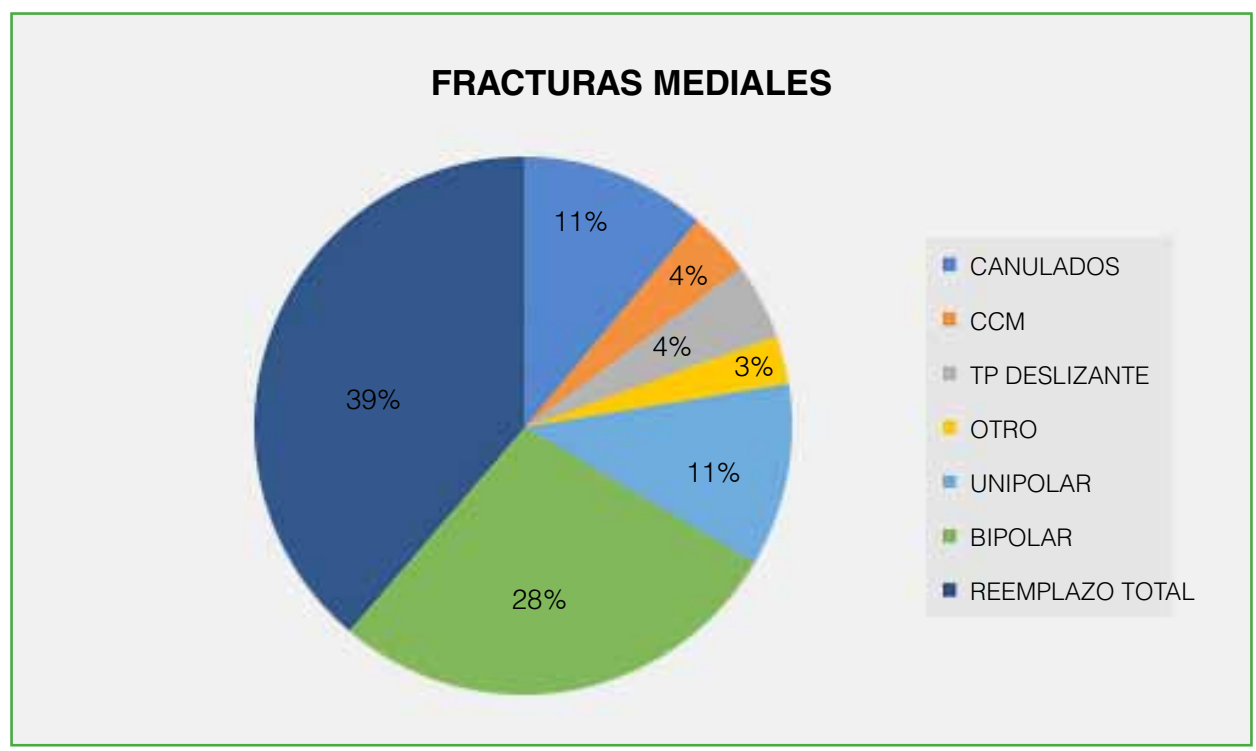

Figura 5. Detalle del tratamiento de las fracturas mediales.

$\mathrm{CCM}=$ clavo cefalomedular, $\mathrm{TP}=$ tornillo placa

En las artroplastias, se adicionó antibiótico al cemento en el 43,14\%. Se usó drenaje en el 52,31\%.

En cuanto a las 505 respuestas para las fracturas laterales, en el 80,2\%, se utilizó una osteosíntesis; en el 19\%, una artroplastia y, en el 0,79\% ( 3 casos), se indicó un tratamiento conservador. El 87,04\% y el 88,54\% de las osteosíntesis y las artroplastias, respectivamente, eran nacionales. En el 69,79\%, se agregó antibiótico al cemento y el uso de drenaje fue del $14,64 \%$.

Los diferentes tipos de tratamientos de las fracturas intertrocantéricas se detallan en la Figura 6. En la Tabla 4, se resumen los porcentajes comparativos de ambos tipos de fracturas y su tratamiento.

\section{Cuándo comenzó la deambulación posoperatoria}

El tiempo se dividió en días, y se halló que la mitad de los pacientes inició la marcha antes del día 5 posoperatorio y un 10\% no deambuló (Figura 7).

La relación entre este ítem y el tipo de tratamiento indicado según la fractura se detallará en el próximo informe.

\section{Hematocrito, hemoglobina y transfusiones posoperatorias}

Entre 903 respuestas sobre el hematocrito y 811 sobre la hemoglobina, los valores promedio de estos registros fueron $29 \%$ (rango) y $10,62 \mathrm{mg} / \mathrm{dl}$ (rango), respectivamente. 


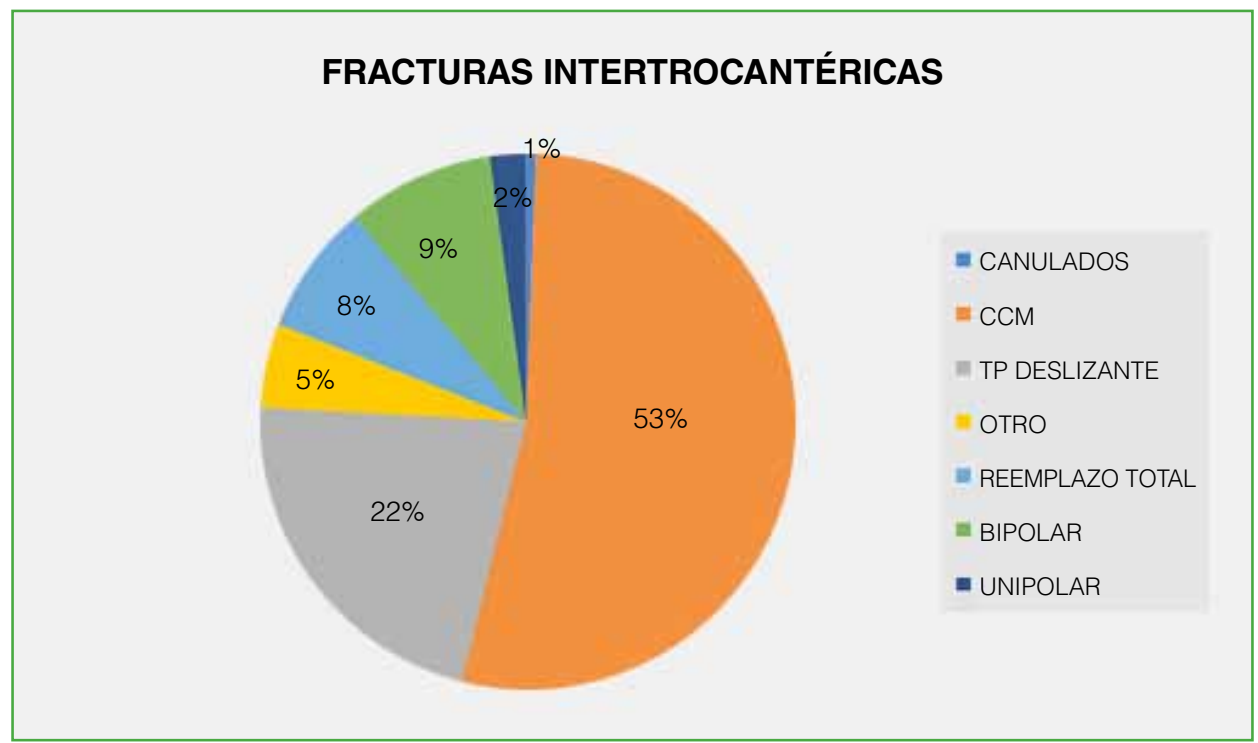

Figura 6. Detalle del tratamiento de las fracturas laterales.

$\mathrm{CCM}=$ clavo cefalomedular, $\mathrm{TP}=$ tornillo placa

Tabla 4. Valores porcentuales comparativos en el tratamiento de las fracturas mediales y laterales

\begin{tabular}{|l|c|c|c|c|c|c|c|c|}
\hline Tratamiento & $\begin{array}{c}\text { Osteo- } \\
\text { síntesis }\end{array}$ & $\begin{array}{c}\text { Artro- } \\
\text { plastia }\end{array}$ & $\begin{array}{c}\text { Clavo cefalo- } \\
\text { medular }\end{array}$ & $\begin{array}{c}\text { Tornillo } \\
\text { placa } \\
\text { deslizante }\end{array}$ & $\begin{array}{c}\text { Tornillo } \\
\text { canulado }\end{array}$ & $\begin{array}{c}\text { Reemplazo } \\
\text { total } \\
\text { de cadera }\end{array}$ & $\begin{array}{c}\text { Prótesis } \\
\text { bipolar }\end{array}$ & $\begin{array}{c}\text { Prótesis } \\
\text { unipolar }\end{array}$ \\
\hline $\begin{array}{l}\text { Fracturas } \\
\text { mediales } \\
n=444\end{array}$ & 22,29 & 77,25 & 4 & 4 & 11 & 39 & 28 \\
\hline $\begin{array}{l}\text { Fracturas } \\
\text { laterales } \\
n=505\end{array}$ & 80,2 & 19 & 53 & 22 & 1 & 8 & 9 \\
\hline
\end{tabular}

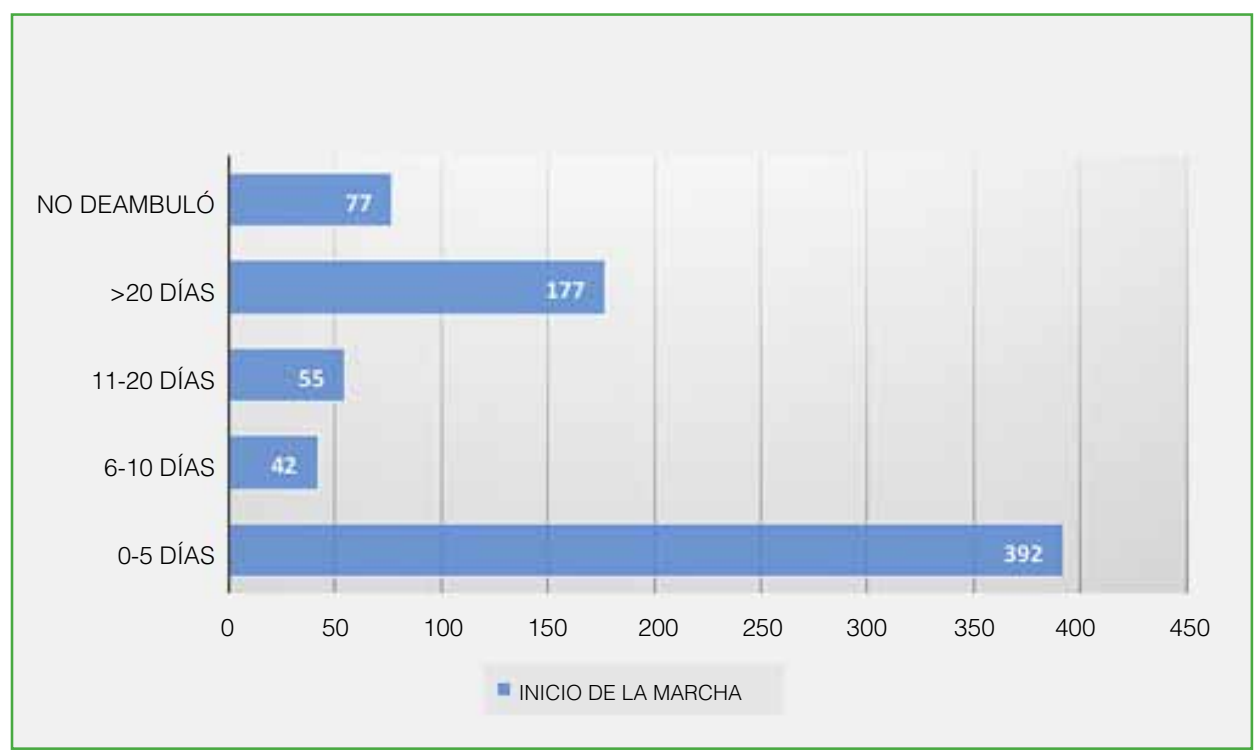

Figura 7. Detalle del día en el que comenzó la marcha posoperatoria. 
Por otro lado, aproximadamente un tercio $(28,8 \%)$ necesitó una transfusión de glóbulos rojos durante el posoperatorio.

\section{Profilaxis antitrombótica}

Hubo 908 respuestas a este ítem. El 95,67\% de los pacientes recibió antitrombóticos durante el posoperatorio, y la enoxaparina fue la droga administrada con más frecuencia (Figura 8).

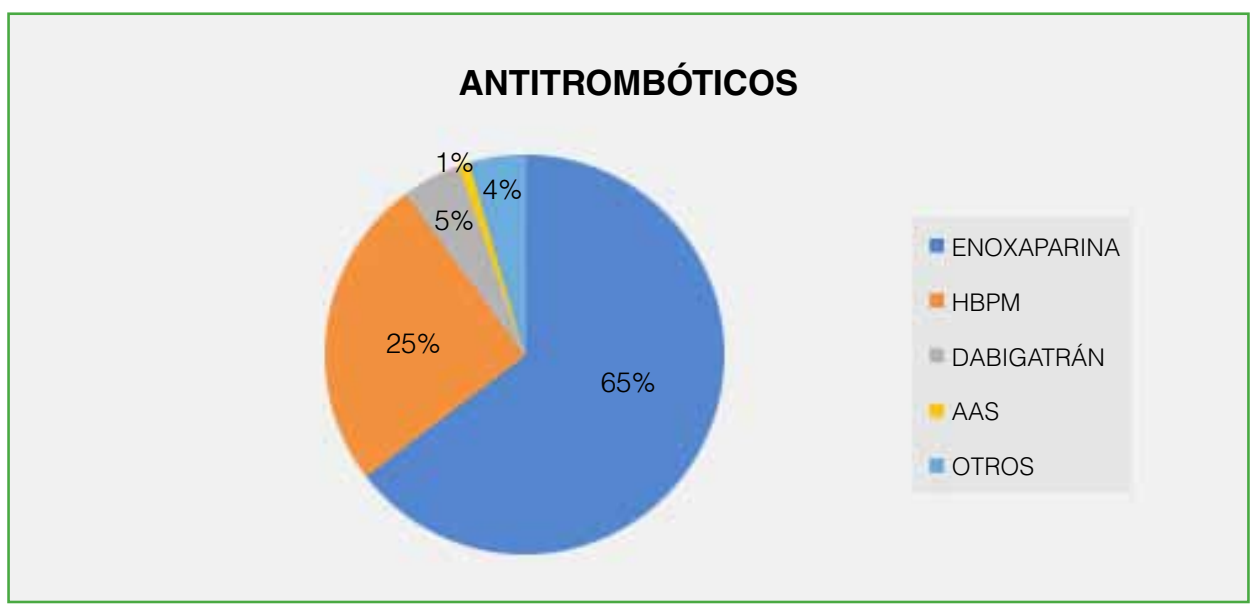

Figura 8. Detalle de las drogas administradas para prevenir complicaciones tromboembólicas. $\mathrm{HBPM}=$ heparina de bajo peso molecular, AAS = ácido acetilsalicílico.

\section{Profilaxis antibiótica}

Según 879 respuestas, los antibióticos más utilizados fueron las cefalosporinas de primera generación (69,62\%) (Figura 9).

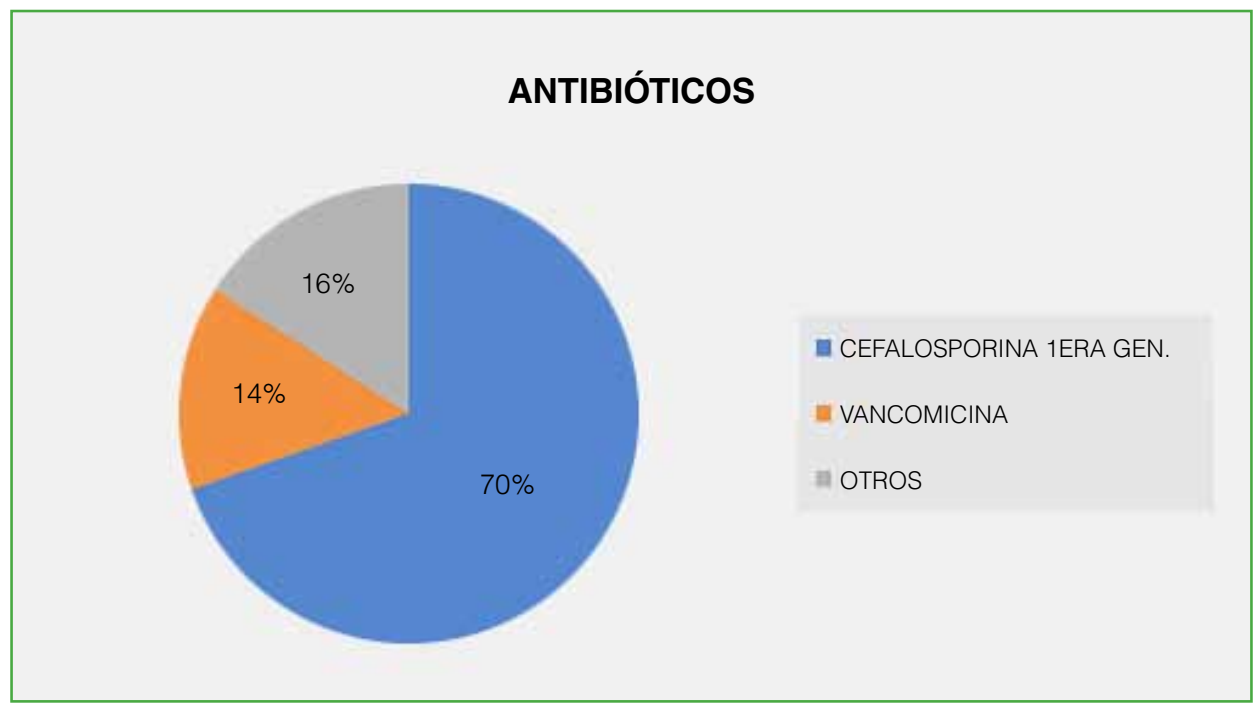

Figura 9. Antibióticos administrados en la profilaxis perioperatoria. (Otros: cefalosporinas, clindamicina, amikacina, colistina, voriconazol, etc.). 


\section{Seguimiento posoperatorio}

Se recibieron 613 respuestas a este ítem. El 45,67\% superó los cuatro meses de seguimiento posoperatorio (Figura 10).

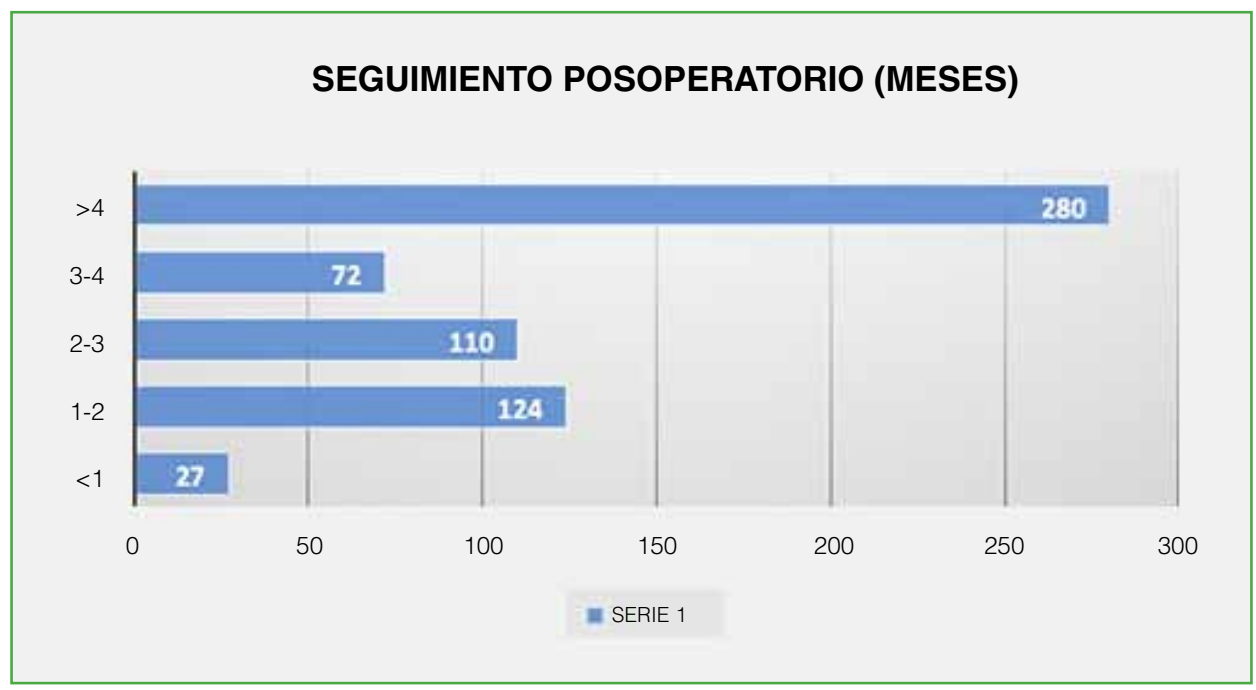

Figura 10. Descripción del seguimiento posoperatorio.

\section{COMPLICACIONES}

Se registraron $121(12,85 \%)$ complicaciones y la más frecuente fue la infección, con una incidencia del 7,27\%. De los 69 casos, 33 correspondieron a infecciones superficiales y 34, a infecciones profundas. En los restantes dos casos, no estaba especificado. La segunda complicación más frecuente fue la luxación de la prótesis, con una incidencia del 3,87\% (17/439 casos).

Se comunicaron complicaciones tromboembólicas (trombosis venosa profunda y tromboembolismo pulmonar) en 12 casos (2\%) (Figura 11).

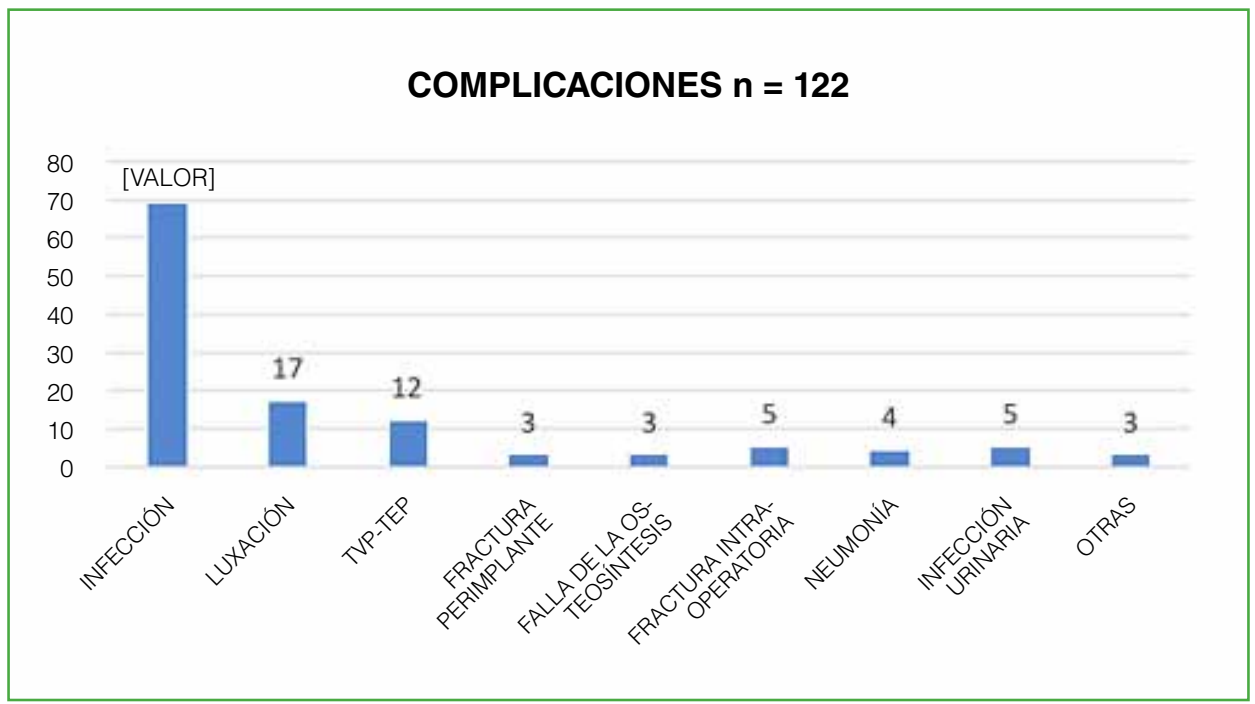

Figura 11. Detalle de las complicaciones registradas.

TVP-TEP $=$ trombosis venosa profunda-tromboembolismo pulmonar 
Hubo cinco fracturas intraoperatorias $(0,82 \%)$, tres posoperatorias ( 2 alrededor de una osteosíntesis y 1 periprotésica) y tres aflojamientos de la osteosíntesis.

El resto de las complicaciones informadas fueron: cinco infecciones del tracto urinario, cuatro neumonías, dos descompensaciones cardíacas y una tromboflebitis en miembros superiores.

\section{Revisiones y reoperaciones}

Se registraron $15(2,46 \%)$ revisiones sobre 608 respuestas específicas para este ítem. En ocho casos, se especificó la causa: tres aflojamientos o pérdidas de fijación de la osteosíntesis, dos luxaciones recidivantes y tres fracturas alrededor del implante. En las restantes siete revisiones, no se detalló el motivo.

Las reoperaciones se relacionaron exclusivamente con las complicaciones infecciosas.

\section{Tasa de mortalidad}

La tasa de mortalidad registrada, dado que, en las encuestas, se detalla específicamente el óbito, fue del 4,85\% (36/741). En el mejor escenario posible, suponiendo que, cuando se omitió la respuesta, el óbito no había sucedido, la tasa de mortalidad sería del 3,8\% (36/949).

Otro dato destacado fue que todos los pacientes que murieron sufrieron alguna complicación detallada en los puntos anteriores.

\section{CONCLUSIONES}

Este análisis descriptivo permite caracterizar a las fracturas de cadera en nuestra población. Estas lesiones afectan predominantemente al sexo femenino (relación mujer:hombre 2,7:1). Alrededor del 80\% tendrá $>70$ años en el momento de la lesión y presentará múltiples comorbilidades (hipertensión arterial, diabetes, osteoporosis, insuficiencia cardíaca, anemia, etc.).

Dos tercios de los afectados vivirán acompañados y cerca del 60\% serán deambuladores dentro del domicilio.

En cuanto al tipo de fractura, no se observa un predominio marcado entre mediales y laterales (47\% vs. 53\%).

La tasa de complicaciones posoperatorias es relativamente elevada, alrededor del 13\%, y la infección es la complicación más frecuente.

Por último, la tasa de mortalidad estimada en los primeros meses posteriores a la cirugía oscilará entre el 3,8\% y el $4,85 \%$.

Continuamos con el análisis de las encuestas, a fin de poder caracterizar estadísticamente, en la segunda parte del informe, los diferentes tipos de tratamientos para cada subtipo de fractura, analizar las relaciones entre las características de la población, las complicaciones y la mortalidad.

Conflicto de intereses: Los autores no declaran conflictos de intereses.

ORCID de J. M. Olivetto: https://orcid.org/0000-0001-9445-0704

ORCID de M. Sierto: https:///rcid.org/0000-0003-3330-0360

ORCID de D. Gamarra: https://orcid.org/0000-0001-7661-850X

\section{BIBLIOGRAFÍA}

1. Clark P, Chico G, Carlos F, Zamudio F, Pereira RM, Zanchetta J, et al. Osteoporosis en América Latina: revisión de panel de expertos. Medware 2013;13(8):e5791. https://doi.org/10.5867/medwave.2013.08.5791

2. Rojas Padilla LG, Quintero Hernández, S, Jimenez Avila J, Lopez Cervantes RE, Amadei R, Pesciallo C, et al. Hip fracture care - Latin America. OTA Int 2020;e064. https://doi.org/10.1097/OI9.0000000000000064

3. Censo Nacional de Población, Hogares y Vivienda 2010. Resultados definitivos. Serie B, No 2, Tomo 1. Argentina, Instituto Nacional de Estadística y Censos (INDEC). Buenos Aires, octubre 2012. http://www.indec.gob.ar 